\title{
Normative data for modified Box and Blocks test measuring upper-limb function via motion capture
}

\author{
Jacqueline S. Hebert, MD, FRCPC $;^{1-2^{*}}$ Justin Lewicke, MBA, BSc; $^{2}$ Thomas R. Williams, PhD, PEng; $^{3}$ Albert \\ H. Vette, $\mathbf{P h D}^{2-3}$ \\ ${ }^{1}$ Division of Physical Medicine and Rehabilitation, University of Alberta, Edmonton, Alberta, Canada; ${ }^{2}$ Glenrose \\ Rehabilitation Hospital, Alberta Health Services, Edmonton, Alberta, Canada; ${ }^{3}$ Department of Mechanical Engineer- \\ ing, University of Alberta, Edmonton, Alberta, Canada
}

\begin{abstract}
Motion analysis is an important tool for examining upper-limb function. Based on previous work demonstrating a modified Box and Blocks (BB) test with motion capture to assess prosthetic performance, we collected data in 16 nondisabled participants to establish normative kinematics for this test. Four motions of the modified BB test were analyzed to establish kinematic data for upper-limb and trunk motion. The test was repeated for right and left arms in standing and seated positions. Data were compared using a nonparametric Friedman test. No differences were found between right- and lefthand performance other than for task completion time. Small but significant differences were found for standing and seated performance, with slightly greater ranges in standing for axial trunk rotation, medial-lateral sternum displacement, and anterior-posterior hand displacement. The kinematic trajectories, however, were very consistent. The consistency in our nondisabled data suggests that normative kinematic trajectories can be defined for this task. This motion capture procedure may add to the understanding of movement in upper-limb impairment and may be useful for measuring the effect of interventions to improve upper-limb function.
\end{abstract}

Key words: biomechanics, Box and Blocks test, kinematics, limb function, motion, motion capture, outcome measures, patient outcome assessment, prosthetic performance, upper limb.

\section{INTRODUCTION}

Upper-limb impairments affect daily living in a wide variety of injury and illness conditions. Interventions aimed at facilitating upper-limb function focus on improving functional performance, improving quality of movement, and reducing compensatory motion. Motion analysis is increasingly being used as a method of quantifying range of joint motion of the upper limb, speed of motion, dexterity, and compensatory movements. Various standardized methods for assessing upper-limb functional tasks have been proposed for defining an upper-limb motion analysis protocol with varying levels of complexity. Most authors have advocated using motions that simulate a common movement activity of daily living (ADL) task, such as drinking, combing hair or reaching hand to head [1-3], jar opening [4], removing a parking token [5], or reaching and placing a ball [6]. In this context, it has

\footnotetext{
Abbreviations: ADL = activity of daily living, AP = anteriorposterior, $\mathrm{BB}=\mathrm{Box}$ and Blocks (test), $\mathrm{C}=$ cervical, $\mathrm{FE}=$ flexion, $\mathrm{LB}$ = lateral bending, $\mathrm{ML}=$ medial-lateral, $\mathrm{RT}=$ rotation, $\mathrm{SD}=$ standard deviation, $\mathrm{T}=$ thoracic.

*Address all correspondence to Jacqueline S. Hebert, MD, FRCPC; University of Alberta, Division of Physical Medicine and Rehabilitation, 5005 Katz Group Centre, Edmonton, Alberta T6G 2E1, Canada; 780-735-8218; fax: 780735-6018. Email: jhebert@ualberta.ca

http://dx.doi.org/10.1682/JRRD.2013.10.0228
} 
been found important to measure not only ranges of upper-limb joint motion but also velocity and dexterity of the motion as an overall indicator of performance [2]. The task may be of a cyclical or noncyclical nature, but when the task is nonconstrained, it can be difficult to assess an intervention effect due to variability in task execution [2] and different strategies used to accomplish the goal of the task [1].

Given the extensive kinematic data that can be collected, extracting clinically meaningful information can be a challenge. Using simple motion tasks with constrained movement patterns, more accurate and reproducible kinematic data may be attained, but the lack of performance demand associated with the task may alter the way the individual executes the task. For this reason, functional and timed measures of performance are often clinically chosen as objective outcome measures for upper-limb impairment rather than constrained motion analysis tasks. However, combining the two methods by encouraging competitive, goal-oriented performance within the constraints of motion capture could possibly help bridge the gap between laboratory and real-world performance.

For this reason, we previously used a modified version of the Box and Blocks (BB) test with motion capture to examine upper-limb prosthetic function and myoelectric control after targeted muscle reinnervation surgery [7]. We chose the BB test because it is a widely used, well-validated timed performance measure of upper-limb function [8] that requires repetitive consistent execution, with good responsiveness to change in performance [9]. We modified the setup of the test for motion analysis to simplify the block selection and order of movement while still allowing repetitive cycles of motion with a distinct performance goal. In Hebert and Lewicke, we demonstrated significant limitations in wrist and elbow motion along with increased trunk compensatory movements required by the prosthetic user [7]. This combination of a performance metric (activity) with kinematic measurements (body functions and structures) assesses two recommended domains of the World Health Organization International Classification on Functioning, Disability, and Health for upper-limb impairment [10]. We therefore sought to define a set of normative kinematic data for this modified BB test to further investigate its utility as an outcome measurement tool for upper-limb impairment.

The goals of this report are to (1) present normative data for a standardized modified version of the BB test using motion analysis to provide information about the movements of the upper limb and the trunk and (2) compare differences between standing and seated positions to determine the validity of administering the test in a standing position compared with the seated position used for the original BB normative data set [8]. By meeting these goals, we aim to present a potential method for standardized assessment of upper-limb motion and function for a variety of upper-limb impairments.

\section{METHODS}

\section{Participants}

Normative data were acquired from 16 nondisabled participants: seven female and nine male, age (mean \pm standard deviation [SD]): $29.5 \pm 8.9$ yr (range: $21-54$ yr), and height: $172.6 \pm 10.7 \mathrm{~cm}$ (range: $154.9-190.5 \mathrm{~cm}$ ). All participants were right-hand dominant and had no upperlimb pathology or history of neurological or musculoskeletal injury.

\section{Modifications of Original Box and Blocks Test}

The BB test was modified from the original version in that, rather than a random array of blocks, 16 blocks were placed in 4 rows with defined placement locations (Figure 1). As per the original test, the blocks were placed on the side of the arm being tested. Each participant was instructed to start with the block farthest away and most lateral (hand position shown in Figure 1) and to proceed across the row to the most medial block, then down to the next row from the outside (most lateral) block inward. This required specific targeting and sequencing of motion. The instructions given to the participant were otherwise similar to the original test description for adults, asking the participant to work as quickly as possible but to ensure their fingers cross the divider as they move the block over it [8]. We collected kinematic data throughout the trial and recorded the time to complete the transfer of blocks, defined from the first motion of block 1 to the first motion of block 16 (i.e., 15 full completion cycles).

Each participant executed four trials: right hand standing, left hand standing, right hand sitting, and left hand sitting. The trials were conducted in the same order for all participants. For all trials, the top of the heightadjustable table was set at the level of the participant's anterior superior iliac spines in both the seated and standing positions. The box was placed at the edge of the table 


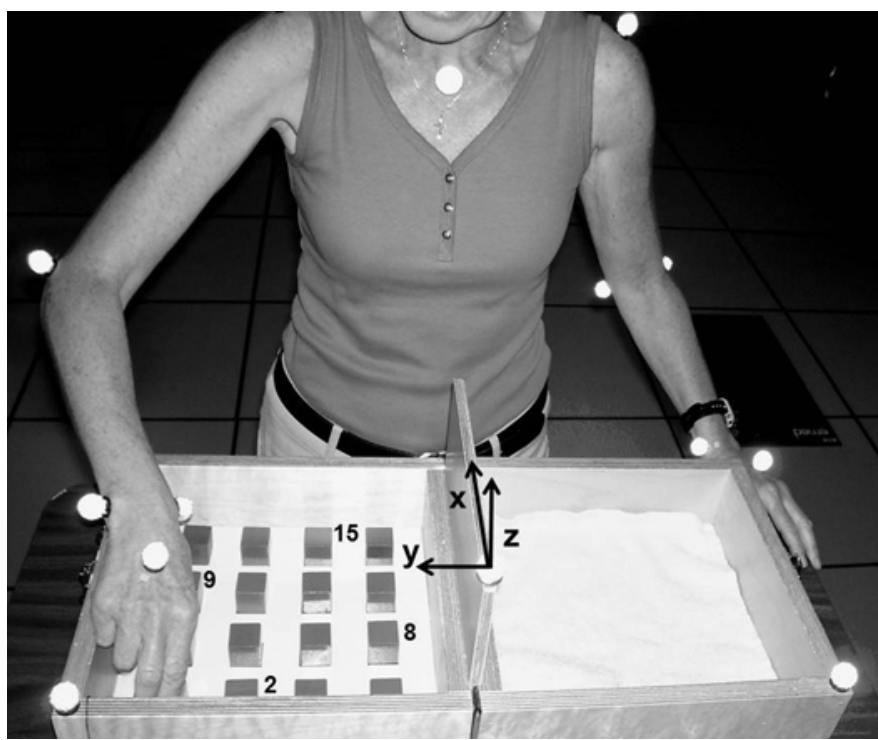

Figure 1.

Setup of modified Box and Blocks test with motion capture. Each participant was instructed to proceed from outer upper-corner block (hand shown on starting block), across row to most medial block, then down to next row from outside block in. Global reference frame for motion capture was attached to marker on top of box divider. Note: $x$-axis points posterior, $y$-axis points right, and $z$-axis points superior to participant.

closest to the participant, with the blocks placed in the grid position on the side of the arm being tested. The participant was asked to rest both hands on the table lateral to the sides of the box at the start of the test and to leave the nontested arm in the same position throughout the test. Instructions were given verbally to the participant, and one initial practice trial was executed to allow familiarization with the task. For the seated trials, the participant was seated in a nonrotating, height-adjustable chair with no arm rests, with the knees and hips at approximately $90^{\circ}$. The participant was asked to leave his or her feet flat on the floor and the hands in the same position as in the standing trials.

\section{Data Collection and Processing}

Motion data were captured using eight optoelectronic cameras that use visible red light to track the trajectories of reflective markers (Motion Analysis Corporation; Santa Rosa, California). Markers were placed on the following anatomical locations: bilateral acromion (most dorsal point on the acromioclavicular joint), sternum (deepest point of incisura jugularis), cervical (C)7 (spinous process, 7th cervical vertebra), thoracic (T)8 (spinous process, 10th thoracic vertebra), lateral elbow (most caudal point on lateral epicondyle), medial elbow (most caudal point on medial epicondyle), lateral wrist (most caudal-lateral point on the radial styloid), medial wrist (most caudal-medial point on the ulnar styloid), and hand (distal end of the 3rd metacarpal phalanx). The two elbow and two wrist markers were used to estimate the elbow and wrist joint centers, respectively (Figure 1). Two markers were also placed on the left and right sides of the box and one on top of the divider in order to identify the location of the box in the virtual laboratory space as well as the participant's location relative to the box. All motion data were captured at a sampling frequency of $60 \mathrm{~Hz}$ and expressed in the global reference frame with origin at the marker on top of the box divider. The $x$-axis pointed posterior, the $y$-axis pointed right, and the $z$-axis pointed superior to the participant (Figure 1).

A range of kinematic parameters were identified. Trunk flexion (FE), lateral bending (LB), and axial rotation (RT) with respect to the global reference frame were computed via the triangle of C7, T8, and the sternum following a previously described methodology [11]. Upperarm FE/extension, adduction/abduction, and axial RT were defined with respect to a trunk reference frame that was aligned with the global reference frame during natural upright standing. Upper-arm orientation was identified via the triangle of lateral elbow, medial elbow, and corrected acromion location following the methodology outlined by Rab et al. [3]. Note that an additional marker at the medial elbow was used to eliminate the possibility of marker collinearity. Pseudo-elbow FE was defined by the angle between the wrist joint center, the elbow joint center, and the acromion [7]. Pseudo-wrist FE was defined by the angle between the dorsal hand marker, the wrist joint center, and the elbow joint center. Anteriorposterior (AP), medial-lateral (ML), and vertical trunk translation were measured as the displacement of the sternum marker with respect to global reference frame. AP, ML, and vertical hand translation were measured by the displacement of the dorsal hand marker with respect to the global reference frame (with origin at the marker on the box divider). Using this definition, negative hand displacements along the longitudinal (frontal) axis indicated the dorsal hand marker being below (to the left of) the divider (e.g., left hand picking up a block), and positive displacements along the longitudinal (frontal) axis 
indicated the dorsal hand marker being above (to the right of) the divider (e.g., left hand dropping a block).

\section{Kinematic Data Analysis}

For both "body postures" and "body sides," the following quantitative measures were identified: (1) trial completion time, (2) range of each kinematic parameter, and (3) maximum absolute velocity of each kinematic parameter. Trial completion time was defined as the time from first motion of block 1 to first motion of block 16 (i.e., 15 full completion cycles) in order to eliminate the effect of reaction time variation at the start of the trial and to utilize a well-defined end point at trial completion. Note that first motion of blocks 1 and 16 was defined as the time when the vertical velocity of the hand marker changed its sign from negative to positive at the beginning of these two block cycles. The Friedman test was applied to all measures to identify significant differences in the group mean between body posture (standing and sitting) and body side (right and left). Note that this nonparametric analysis of variance equivalent was used because we cannot be certain that identified measures are normally distributed.
In addition to the quantitative measures, we also determined normative trajectories for kinematic parameters and four specific block cycles. Similar to gait analysis conventions [12], trajectories were normalized with respect to block cycle $(0 \%-100 \%)$ in order to eliminate the effect of completion speed for the trajectories. Blocks 2, 8, 9, and 15 were selected for trajectory analysis in order to represent all rows and columns of the block placement grid as well as "near-far" and ML reaching quadrants (Figure 1).

\section{RESULTS}

The Friedman test with factors body side and body posture revealed that trial completion time was significantly less for right-hand performance than left-hand performance ( $p=0.01)$. Since none of the other quantitative measures exhibited significant differences between rightand left-hand performance ( $p>0.05)$, they were combined post hoc for the two body sides (for both body postures separately). The Table presents all measures for the

Table.

Quantitative measures for standing and sitting performance.

\begin{tabular}{|c|c|c|c|c|}
\hline Measure & \multicolumn{2}{|c|}{ Standing (s) } & \multicolumn{2}{|c|}{ Sitting (s) } \\
\hline \multicolumn{5}{|l|}{ Trial Completion Time } \\
\hline Right Hand (blocks 1-15) & \multicolumn{2}{|c|}{$9.65 \pm 0.86$} & \multicolumn{2}{|c|}{$9.70 \pm 0.90$} \\
\hline \multirow[t]{3}{*}{ Left Hand (blocks 1-15) } & \multicolumn{2}{|c|}{$10.36 \pm 1.12$} & \multicolumn{2}{|c|}{$10.38 \pm 1.18$} \\
\hline & \multicolumn{2}{|c|}{ Range ( $\left.{ }^{\circ}\right)$} & \multicolumn{2}{|c|}{ Maximum Absolute Velocity ( $\%$ /s) } \\
\hline & Standing & Sitting & Standing & Sitting \\
\hline \multicolumn{5}{|l|}{ Kinematic Parameter } \\
\hline AP Trunk Angle & $5.0 \pm 2.1$ & $4.9 \pm 2.3$ & $27.0 \pm 15.1$ & $24.6 \pm 10.5$ \\
\hline ML Trunk Angle & $5.2 \pm 1.9$ & $5.3 \pm 2.2$ & $38.2 \pm 12.1$ & $33.9 \pm 11.1$ \\
\hline RT Trunk Angle & $10.3 \pm 2.9$ & $8.7 \pm 1.9$ & $56.3 \pm 15.5$ & $52.9 \pm 12.0$ \\
\hline Upper-Arm FE & $36.1 \pm 6.4$ & $34.2 \pm 6.3$ & $203.1 \pm 37.7$ & $184.1 \pm 43.5$ \\
\hline Upper-Arm AA & $23.0 \pm 5.0$ & $24.4 \pm 5.8$ & $149.6 \pm 40.1$ & $162.1 \pm 43.6$ \\
\hline Upper-Arm RT & $42.3 \pm 8.8$ & $39.8 \pm 8.7$ & $383.4 \pm 82.3$ & $334.0 \pm 79.5$ \\
\hline Elbow Angle & $45.3 \pm 7.2$ & $43.0 \pm 7.8$ & $333.7 \pm 100.3$ & $297.8 \pm 66.4$ \\
\hline \multirow[t]{3}{*}{ Wrist Angle } & $32.6 \pm 7.7$ & $29.5 \pm 7.8$ & $435.8 \pm 169.3$ & $372.8 \pm 160.6$ \\
\hline & \multicolumn{2}{|c|}{ Range (mm) } & \multicolumn{2}{|c|}{ Maximum Absolute Velocity (mm/s) } \\
\hline & Standing & Sitting & Standing & Sitting \\
\hline \multicolumn{5}{|l|}{ Kinematic Parameter } \\
\hline AP Sternum Displacement & $32.4 \pm 16.1$ & $33.7 \pm 13.3$ & $97.7 \pm 34.4$ & $92.9 \pm 23.4$ \\
\hline ML Sternum Displacement & $44.2 \pm 21.9$ & $32.6 \pm 11.9$ & $146.6 \pm 51.1$ & $136.7 \pm 24.7$ \\
\hline Vertical Sternum Displacement & $11.4 \pm 5.0$ & $12.2 \pm 4.9$ & $60.2 \pm 22.6$ & $60.0 \pm 17.9$ \\
\hline AP Hand Displacement & $154.0 \pm 11.7$ & $147.0 \pm 12.8$ & $686.2 \pm 214.9$ & $668.7 \pm 174.6$ \\
\hline ML Hand Displacement & $261.5 \pm 44.5$ & $250.1 \pm 43.1$ & $1,581.9 \pm 354.4$ & $1,431.3 \pm 297.2$ \\
\hline Vertical Hand Displacement & $215.8 \pm 19.4$ & $211.4 \pm 21.0$ & $1,507.0 \pm 241.1$ & $1,492.1 \pm 255.9$ \\
\hline \multicolumn{5}{|c|}{$\begin{array}{l}\text { Note: Shown are trial completion time, range of each kinematic parameter, and maximum absolute velc } \\
\text { tion). Except for completion time }(n=16 \text { and } 16) \text {, measures are combined for right- and left-hand perfo } \\
\text { measures for two postures are shown in bold }(p<0.05) \text {. } \\
\text { AA = adduction/abduction, AP = anterior-posterior, FE = flexion, ML = medial-lateral, RT = rotation. }\end{array}$} \\
\hline
\end{tabular}


standing and sitting conditions. When comparing the standing performance with the seated performance, significantly larger values during standing were found for three variables (bold numbers, Table): trunk RT range $(p=0.01)$, ML sternum displacement range $(p=0.01)$, and AP hand displacement range $(p=0.01)$.

Based on the fact that (1) kinematic trajectories were time-normalized and (2) all kinematic parameters were not significantly different between right- and left-hand performance, trajectories were combined post hoc for the two body sides. Figures 2 to 7 depict the trajectories of the trunk angles, upper-arm angles, elbow FE, wrist FE, sternum displacement, and hand displacement for the standing trials. For each figure, trajectories for blocks 2 (far-lateral block), 8 (far-medial block), 9 (near-lateral block), and 15 (near-medial block) are shown. Note that trajectories during right-hand performance were transformed to be in agreement with left-hand performance (ML and RT as well as ML displacements). The solid thin lines mark the mean and the dashed thin lines mark the SD of the trajectories for all trials $(n=2 \times 16)$. Due to intermittent occlusion of wrist markers, the normative wrist FE trajectories were based on a reduced number of trials (Figure 5, $n \geq 13$ ). Note that for kinematic parameters that were shown to be significantly different in the seated position (bold numbers, Table), the seated trajectories were plotted on the corresponding standing trajectory graphs for visual comparison (Figures 2, 6, and 7).

Figure 2 shows a very narrow range of trunk FE and LB for all block cycles. As might be expected, trunk RT at the start of the block cycle for far blocks 2 and 8 (approximately $-5^{\circ}$ of RT) was slightly greater than the more neutral trunk position at the start of cycle for near blocks 9 and 15. Note that although axial trunk RT was significantly different for standing versus sitting (Table), the mean kinematic trajectory for the sitting trial (bold dashed line, Figure 2) still fell within \pm 1 SD of the respective trajectory during standing.

As expected, Figure 3 shows reduced upper-arm FE at the start of the block cycle for near blocks 9 and 15 compared with the start of the block cycle for far blocks 2 and 8, with the greatest amount of arm FE required for the far lateral block $\left(51^{\circ}\right.$, compared with $33^{\circ}$ for the near medial block). The graphs also show that while the greatest amount of upper-arm FE was required for far lateral block 2, a greater degree of upper-arm abduction was required at the start of the block cycle for near-lateral block 9 .
Figure 4 shows that far-lateral block 2 required the greatest amount of elbow range, requiring on average between $77^{\circ}$ and $111^{\circ}$ of elbow FE throughout the block cycle. Wrist FE trajectories (Figure 5) were fairly consistent across the blocks, although near-medial block 15 seemed to have a tighter SD, likely due to being an inside corner block with more limited access due to the box edges.

In Figure 6, sternum displacement parallels the trunk trajectories of Figure 2, with a fairly narrow range. Although the ML sternum displacement range was significantly different between the two body postures (Table), the mean kinematic trajectory for the sitting trial (bold dashed line, Figure 6) was very close to the one during standing. The AP and ML hand trajectories in Figure 7 reflect the start and end positions of the specified blocks, and the vertical hand motion shows a smooth trajectory with consistent curve and height crossing over the divider. Although the AP hand displacement range was significantly different between the two body postures (Table), the mean kinematic trajectory for the sitting trial (bold dashed line, Figure 7) fell within \pm 1 SD of the respective trajectory during standing.

\section{DISCUSSION}

The main objective of this study was to present adult normative data for a standardized modified version of the $\mathrm{BB}$ test using motion analysis to provide information about the movements of the upper limb and trunk. We chose to use the BB test because it is widely used clinically as a functional performance metric. The BB test has strong clinical utility in the assessment of neurologic impairment [13], musculoskeletal impairment [14], and in the elderly [15]. It provides information on performance (number of blocks moved as a proxy of speed) but not on how the body is actually moving. Adding the examination of motion capture kinematics to this functional performance test can enhance the understanding of the movements required to accomplish the task. This could provide evidence on whether the patient is improving by learning compensatory motions or by improving normal movement patterns. In this way, analyses with motion capture could give insight into how the patient has improved.

Before applying a new standardized metric to impaired populations, it is important to first define the 

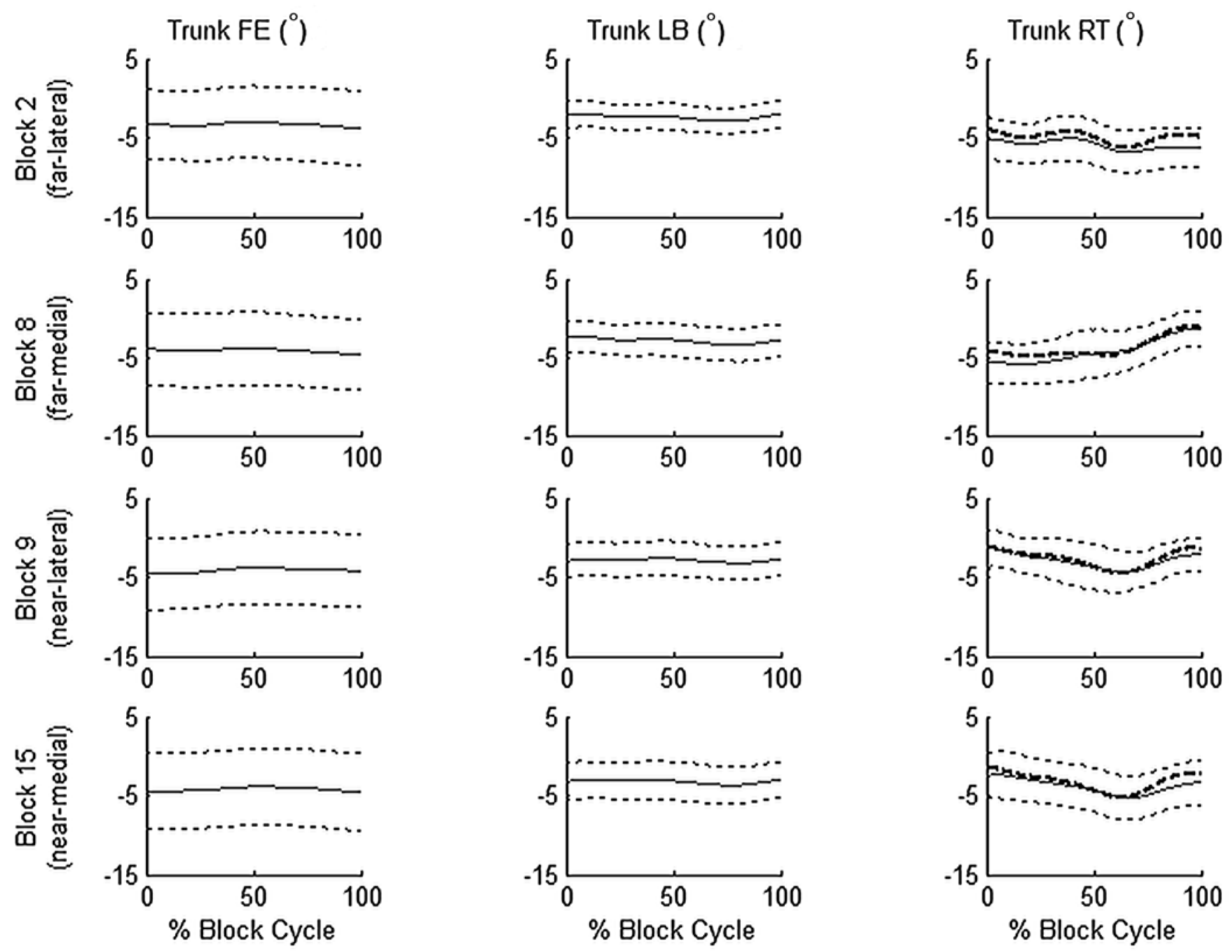

Figure 2.

Trunk angle trajectories for normative sample in standing position. Solid thin lines mark mean and dashed thin lines mark standard deviation of trajectories for blocks 2, 8, 9, and 15 for all trials. Trajectories during right-hand performance were transformed to be in agreement with left-hand performance (flexion [FE], lateral bending [LB], and rotation [RT]). Because range of axial trunk RT was significantly different between two body postures (Table), trunk RT plots also depict average trajectories for sitting position (bold dashed line).

consistency of performance and kinematics in nondisabled participants. Even with our relatively small sample size, there was good consistency in our normative data with small variation from the mean, indicating consistent performance between participants without upper-limb impairment. This is in agreement with other studies examining constrained movements of the arm [2] and unconstrained upper-limb standardized tasks [1] that have also been able to demonstrate characteristic trajectories with small SDs in as few as 10 nondisabled participants. This suggests that there is a relatively tight standard range and technique used by nondisabled participants to perform defined upper-limb tasks.

We chose to control the block selection of the BB test with a specific protocol and to analyze specific block movements representative of near-far and ML extremes (Figure 1). This approach simplified the kinematic graphs in comparison with plotting the entire 16 block 

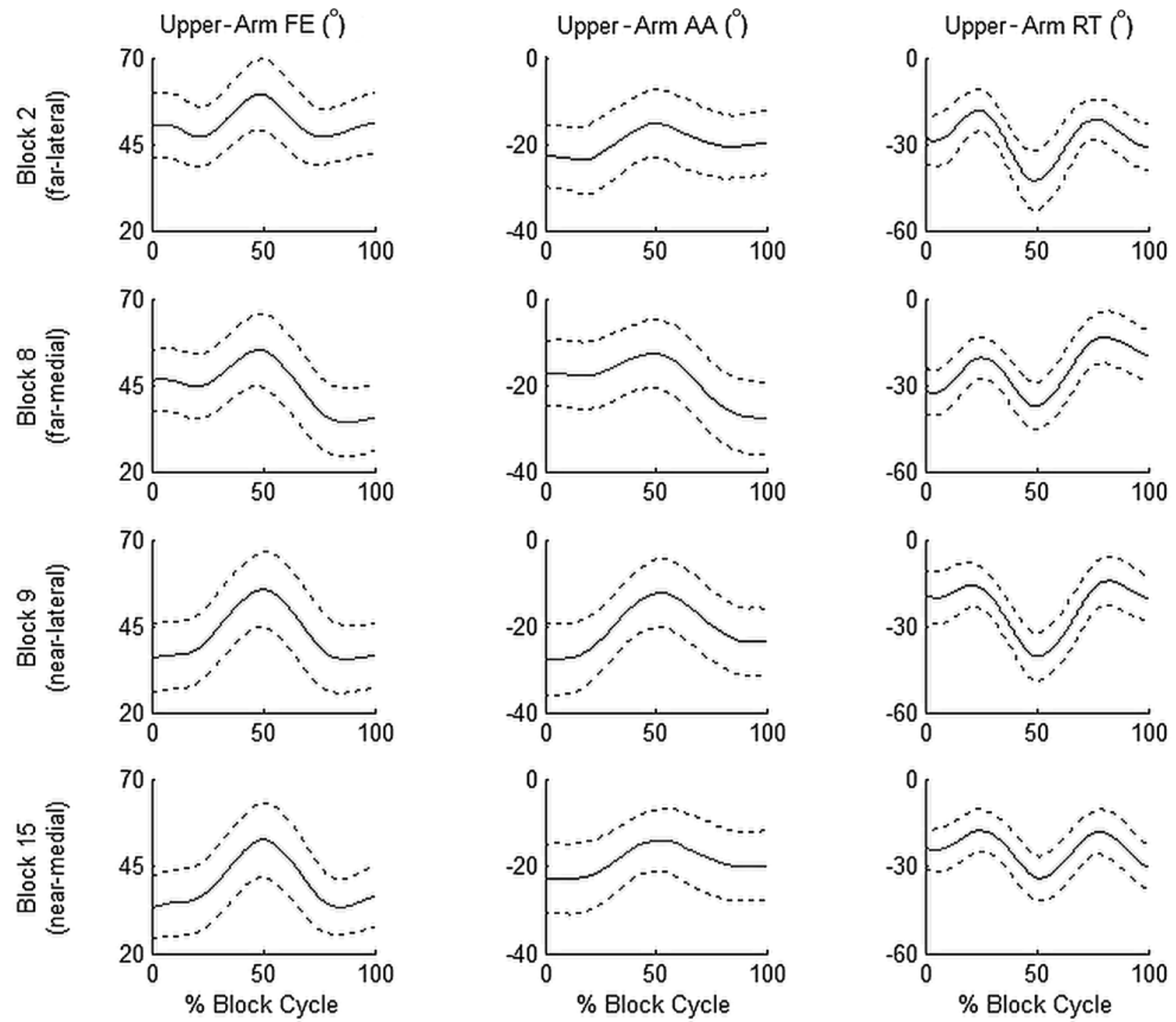

Figure 3.

Upper-arm angle trajectories for normative sample in standing position. Solid thin lines mark mean and dashed thin lines mark standard deviation of trajectories for blocks 2, 8, 9, and 15 for all trials. Trajectories during right-hand performance were transformed to be in agreement with left-hand performance (flexion [FE], adduction/abduction [AA], and rotation [RT]).

trial. The well-defined start and end positions also reduced start- and end-point variability, which has been found to be difficult to control in other upper-limb motion analysis studies [1,4], including our previous study of the BB test in a prosthetic user [7]. Isolating specific block-to-block motions, not inclusive of the first or last block, removed this variability.

The blocks we chose to analyze were representative of different workspace reaching requirements. The assumption was made that participants with different upper-limb impairments might have varying levels of difficulty depending on where they are reaching and that choosing four blocks at different locations would improve the sensitivity of the test. For example, the block closest to the participant near the midline (block 15, Figure 1) might be expected to be difficult to reach with restricted wrist motion due to the need to position the hand to clear the box edge while grasping the block. The wrist FE range required in normal participants when reaching this closest midline block has been noted to be particularly difficult in 
JRRD, Volume 51, Number 6, 2014
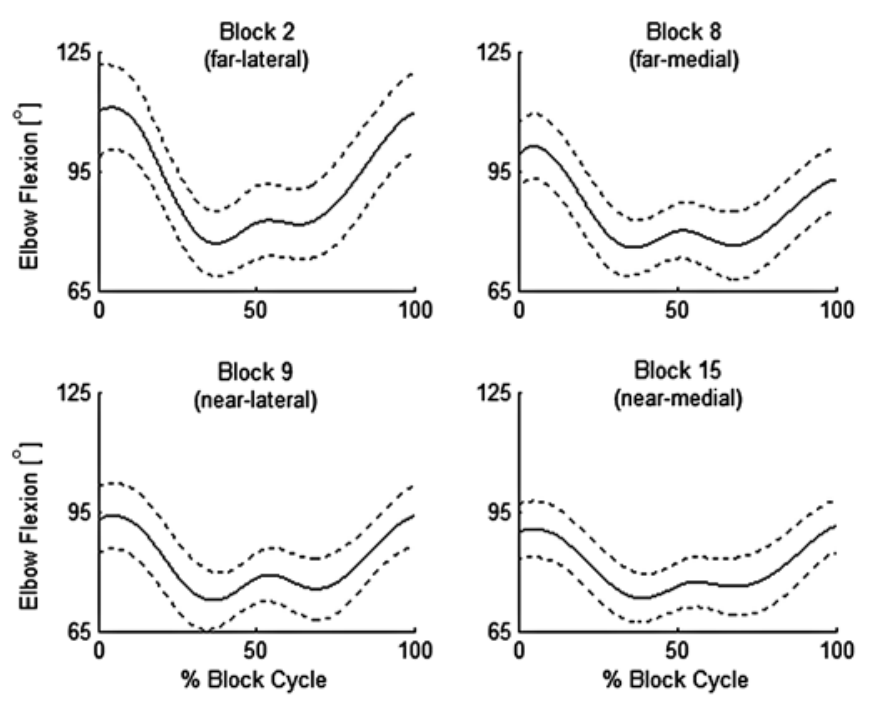

Figure 4.

Elbow flexion trajectories for normative sample in standing position. Solid thin lines mark mean and dashed thin lines mark standard deviation of trajectories for blocks 2, 8, 9, and 15 for all trials.
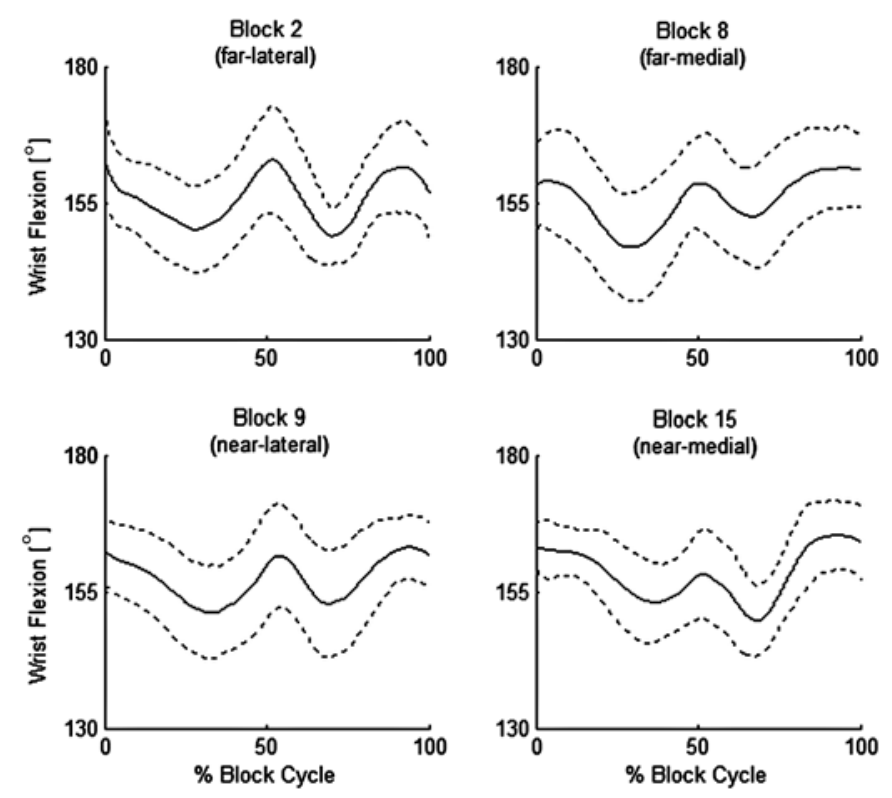

Figure 5.

Wrist flexion trajectories for normative sample in standing position. Solid thin lines mark mean and dashed thin lines mark standard deviation of trajectories for blocks 2, 8, 9, and 15 for all trials $(n \geq 13)$. prosthetic users with no active prosthetic wrist FE [7]. The double peak shape of the wrist FE graphs in our nondisabled participants (Figure 5), reflecting the motions required to pick, lift, release, and then position the hand for grasping the next block, is expected to show significant variation in impaired populations with restricted wrist motion. In comparison, the farthest lateral block (block 2) required the largest amount of elbow extension at the beginning of the cycle (Figure 4) and therefore might be expected to be more difficult in populations with impaired reach function due to elbow motion restrictions. This would be consistent with the finding of limited elbow motion for single reach tasks previously shown in patients with hemiplegic stroke [16] and the synergy noted between trunk FE and reduced elbow motion in reaching tasks in congenital hemiplegia [6].

With respect to the motions of the upper arm, the amount of shoulder FE required at the start of the block cycle is also greatest for the blocks farthest away (block 2, Figure 3). However, because the maximum FE magnitude was relatively constant across the blocks, representing the highest point of hand trajectory when the participant is clearing the block over the divider, the total range required for the block cycle is actually greatest for the nearest block (block 15). This contrasts with the abduction angle being largest for the most lateral block (block 9). These particular graphs would be very interesting to compare with populations with shoulder restrictions, e.g., upper-limb paralysis, who would be expected to have restricted patterns of motion with reaching [16]. Further investigations in impaired populations are needed to confirm these assumptions.

The second objective of our study was to compare differences between standing and seated positions. Although a seated position was used for the original BB test normative data set [8], the test has been reportedly used in the standing position in prosthetic users [17-19]. We chose to normalize the height of the table relative to the pelvis for both positions to reduce the effect of shoulder compensation that would be expected if the relative table height were higher when the participant was seated.

The seated position showed slight differences with less trunk motion and less hand displacement in the AP plane, which could be explained by the fixation of the seated pelvis in relation to the table because participants were asked to sit at a comfortable distance at the start of the trial without being able to alter their position. For the standing position, participants could vary their position 

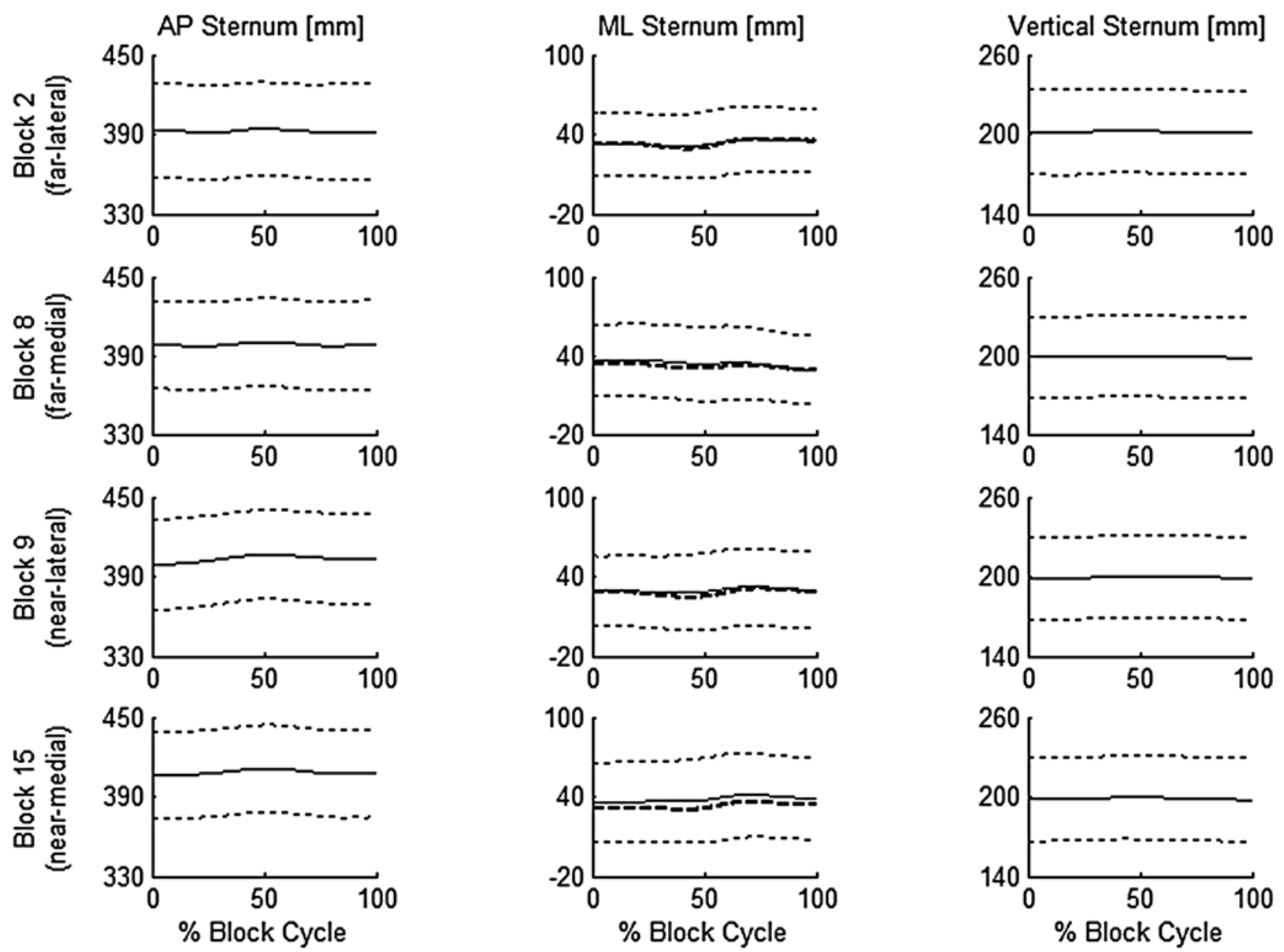

Figure 6.

Three-dimensional displacement trajectories of sternum for normative sample in standing position. Solid thin lines mark mean and dashed thin lines mark standard deviation of trajectories for blocks 2, 8, 9, and 15 for all trials. Trajectories during right-hand performance were transformed to be in agreement with left-hand performance (medial-lateral [ML] displacements). Because range of $\mathrm{ML}$ sternum displacement was significantly different between two body postures (Table), ML sternum displacement plots also depict average trajectories for sitting position (bold dashed lines). AP = anterior-posterior.

throughout the trial, e.g., shifting their weight, potentially encouraging more trunk motion. However, the differences in the parameters for the seated posture were quite small and did not substantially deviate from the mean kinematic trajectories during standing. This suggests that the test has equal validity in sitting and standing with regard to kinematics, so long as the slightly restricted trunk RT is taken into account in sitting. Trunk motion is important to consider with reaching tasks in impaired participants, because less elbow extension and supination to reach for objects is commonly compensated for by increased trunk FE [20]. Increased trunk motion is typically seen during gross motor functions and cyclical reaching tasks in children with hemiplegic cerebral palsy $[6,20]$ and can be presumed to be important with most upper-limb impairments restricting range of motion.

In our protocol, the presentation of trials was not randomized, and this might introduce a possible bias due to learning effect. However, each participant performed only four trials, and right-hand performance was faster than 

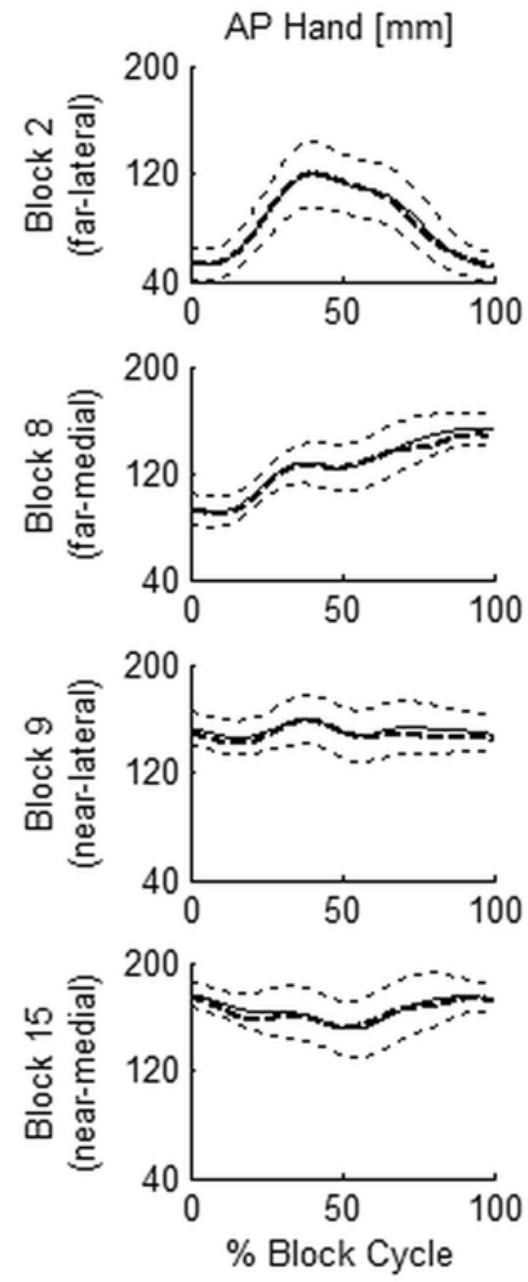
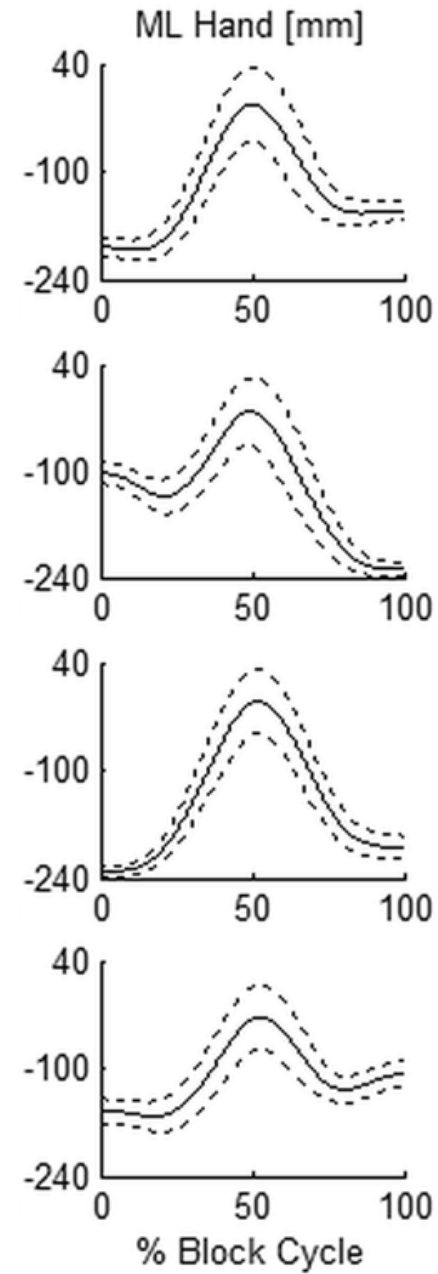
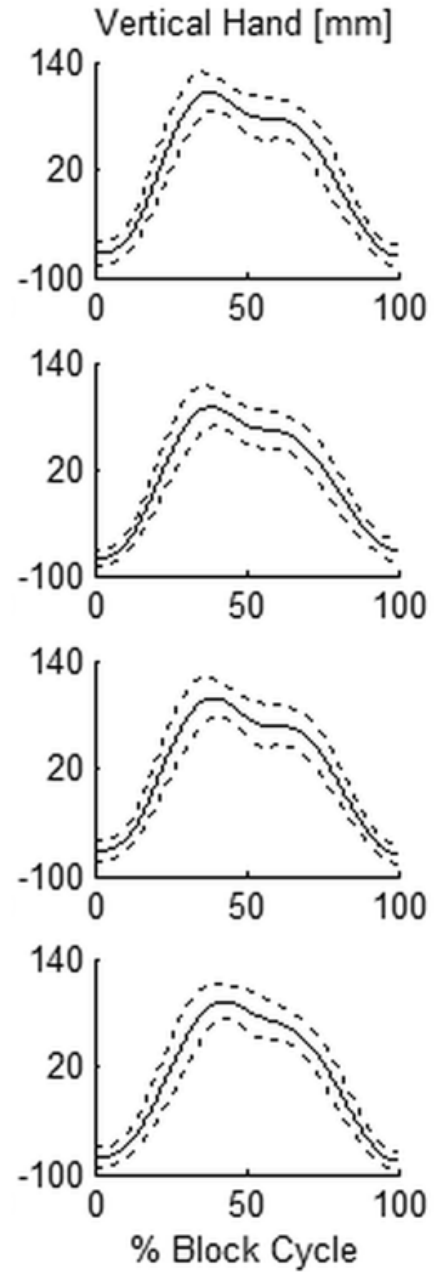

Figure 7.

Three-dimensional displacement trajectories of hand for normative sample in standing position. Solid thin lines mark mean and dashed thin lines mark standard deviation of trajectories for blocks 2, 8, 9, and 15 for all trials. Trajectories during right-hand performance were transformed to be in agreement with left-hand performance (medial-lateral [ML] displacements). Because range of anterior-posterior (AP) hand displacement was significantly different between two body postures (Table), AP hand displacement plots also depict average trajectories for sitting position (bold dashed lines).

left-hand performance despite being performed first, which suggests that hand dominance played a much greater role than learning over this short duration of time. This difference in task completion time between the right and left hands is consistent with the results of Mathiowetz et al., who showed an average performance difference between the hands of $1.5 \mathrm{~s}$ over a $60 \mathrm{~s}$ trial [8]. Our modified abbreviated 16 block trial took around $10 \mathrm{~s}$ for nondisabled participants to complete, which was still long enough to record significant differences between the sides. There was no significant improvement in completion time in seated trials, which were done last, providing further evidence that the learning effect was minimal in our nondisabled participants. However, in impaired populations, the learning effect may be an important factor to consider because functional performance would be expected to improve with repeated practice as part of a treatment protocol. Using this test pre- and posttreatment might help with the interpretation of whether or not abnormal compensatory motions are being utilized to 
speed performance or whether the kinematics of the motion are improving parallel to the performance improvement.

Our protocol is different than other normative motion capture protocols in adult populations that have examined range of motion during ADL tasks [4,21] or a set of tasks simulating ADLs [1-2]. The advantage of task protocols that require a controlled motion task without a specific performance goal or function, such as reaching to the head, is that they result in consistent kinematics from trial to trial [1-2]. However, it is questionable whether this would be representative of real-world performancethat is, would a person brush his or her hair at home using the same controlled reach motion he or she demonstrates in the laboratory under supervision? The advantage of more complex free-motion tasks such as jar opening [4], grooming, and self-care tasks [21] is that they may be more representative of real-world performance. However, they are more complex to analyze and have more variability from trial to trial, particularly at the start and end points of the task [4]. This has led to the recommendation that tasks for upper-limb motion analysis be more cyclical in nature, with an explicit start and finish point, a standard protocol, and a common sequence of events [4]. Indeed, further study by Murgia et al. using a more constrained page-turning task showed more consistent kinematics based on the cyclic nature of the activity [22].

An additional consideration when choosing a task for motion capture is the motivation factor-the participant may very carefully follow the instructions or demonstration in the laboratory, but in the real world, they may choose to accomplish a task the quickest way possible. Letting the participants know they are being timed and asking them to work as quickly as possible adds a performance expectation that is more likely to draw out compensatory movements that would be observed with distractions in the real world. The modified BB test offers a solution between the extremes of the two illustrated approaches - to have the person focus on performance and speed to accomplish the task but have the task goal directed with a standard protocol and specific start and end points. This consistency should allow comparison with normative function and allow detection of change in performance over time. Motion capture with the modified BB test could provide "target" desired kinematic graphs and a standard protocol to evaluate change with intervention, similar to using standard lower-limb kinematic trajectories for gait analysis. It should be noted that, in addition to the kinematic trajectories, the temporal effects are still taken into account by timing task completion and both need to be considered in the interpretation of the results.

\section{LIMITATIONS}

We chose not to examine extensive shoulder and scapular movement, as has been proposed by previous authors [1], due to the difficulty in accessing the scapula with skin mounted markers, as noted by other authors [23]. We chose to use a minimal marker set for providing the information required to make clinical decisions on upper-limb motion. Part of this is the practicality that many of our potential patients are prosthetic users with harnessing and straps that obscure the scapular marker placement, and many clinicians see the extensive marker set as a barrier to bringing their patient in for assessment. A second limitation of our data set was that, for a number of trials, the medial wrist marker was partially obscured by the box divider (mostly for medially located blocks), which limited the data set for wrist kinematics. A larger sample size may have helped to increase the number of trials included in the analysis. Lastly, the proposed test protocol does require motion capture capability similar to that required for quantitative gait analysis, which limits the application to centers with access to the required technology and personnel to administer and interpret the results.

\section{CONCLUSIONS}

We present normative data for a modified BB test using motion capture to study upper-limb and trunk motion. We propose that our method provides a standardized protocol with small variability in nondisabled participants and normative kinematic trajectories representing a range of workspace-level reaching requirements. The protocol may allow assessment of a variety of upper-limb impairments, can be administered standing or seated, and is complementary to currently used outcome measures of upper-limb function by adding motion capture to a common performance task requirement: manipulation, grasp, and relocation of objects at workspace level. This motion capture procedure may add to the understanding of movement in upper-limb impairment and, 
with further study, may be useful for measuring the effect of interventions to improve upper-limb function.

\section{ACKNOWLEDGMENTS}

\section{Author Contributions:}

Study concept and design: J. S. Hebert, J. Lewicke.

Acquisition of data: J. Lewicke, T. R. Williams.

Analysis and interpretation of data: T. R. Williams, A. H. Vette,

J. S. Hebert.

Drafting of manuscript: J. S. Hebert.

Critical revision of manuscript for important intellectual content:

A. H. Vette, J. S. Hebert.

Financial Disclosures: The authors have declared that no competing interests exist.

Funding/Support: This material was based on work supported by the Syncrude Center for Motion and Balance, Glenrose Rehabilitation Hospital, Alberta Health Services.

Institutional Review: All participants gave written informed consent after receiving a detailed explanation about the purposes, benefits, and risks associated with the study. The procedures used in this study were approved by the Health Research Ethics Research Board at The University of Alberta, Edmonton, Canada.

Participant Follow-Up: The authors have no plans to notify the study subjects of the publication of this article because personal and contact information was not retained as per health ethics board approval.

\section{REFERENCES}

1. van Andel CJ, Wolterbeek N, Doorenbosch CA, Veeger DH, Harlaar J. Complete 3D kinematics of upper extremity functional tasks. Gait Posture. 2008;27(1):120-27. [PMID:17459709]

http://dx.doi.org/10.1016/j.gaitpost.2007.03.002

2. Chen W, Xiong C, Huang X, Sun R, Xiong Y. Kinematic analysis and dexterity evaluation of upper extremity in activities of daily living. Gait Posture. 2010;32(4):475-81. [PMID:20692160]

http://dx.doi.org/10.1016/j.gaitpost.2010.07.005

3. Rab G, Petuskey K, Bagley A. A method for determination of upper extremity kinematics. Gait Posture. 2002;15(2): 113-19. [PMID:11869904] http://dx.doi.org/10.1016/S0966-6362(01)00155-2

4. Murgia A, Kyberd PJ, Chappell PH, Light CM. Marker placement to describe the wrist movements during activities of daily living in cyclical tasks. Clin Biomech (Bristol, Avon). 2004;19(3):248-54. [PMID:15003339]

http://dx.doi.org/10.1016/j.clinbiomech.2003.11.012

5. Williams S, Schmidt R, Disselhorst-Klug C, Rau G. An upper body model for the kinematical analysis of the joint chain of the human arm. J Biomech. 2006;39(13):2419-29.

\section{[PMID:16159659]}

http://dx.doi.org/10.1016/j.jbiomech.2005.07.023

6. Steenbergen B, Meulenbroek RG. Deviations in upper-limb function of the less-affected side in congenital hemiparesis.

Neuropsychologia. 2006;44(12):2296-2307.

[PMID:16797611]

http://dx.doi.org/10.1016/j.neuropsychologia.2006.05.016

7. Hebert JS, Lewicke JJ. Case report of modified Box and Blocks test with motion capture to measure prosthetic function. J Rehabil Res Dev. 2012;49(8):1163-74.

[PMID:23341309]

http://dx.doi.org/10.1682/JRRD.2011.10.0207

8. Mathiowetz V, Volland G, Kashman N, Weber K. Adult norms for the Box and Block Test of manual dexterity. Am J Occup Ther. 1985;39(6):386-91. [PMID:3160243] http://dx.doi.org/10.5014/ajot.39.6.386

9. Lin KC, Chuang LL, Wu CY, Hsieh YW, Chang WY. Responsiveness and validity of three dexterous function measures in stroke rehabilitation. J Rehabil Res Dev. 2010; 47(6):563-71. [PMID:20848369]

http://dx.doi.org/10.1682/JRRD.2009.09.0155

10. Metcalf C, Adams J, Burridge J, Yule V, Chappell P. A review of clinical upper limb assessments within the framework of the WHO ICF. Musculoskeletal Care. 2007;5(3): 160-73. [PMID:17610309]

http://dx.doi.org/10.1002/msc.108

11. Vette AH, Yoshida T, Thrasher TA, Masani K, Popovic MR. A comprehensive three-dimensional dynamic model of the human head and trunk for estimating lumbar and cervical joint torques and forces from upper body kinematics. Med Eng Phys. 2012;34(5):640-49. [PMID:22209756] http://dx.doi.org/10.1016/j.medengphy.2011.11.023

12. Kerrigan DC, Todd MK, Della Croce U. Gender differences in joint biomechanics during walking: Normative study in young adults. Am J Phys Med Rehabil. 1998; 77(1):2-7. [PMID:9482373] http://dx.doi.org/10.1097/00002060-199801000-00002

13. Connell LA, Tyson SF. Clinical reality of measuring upperlimb ability in neurologic conditions: A systematic review. Arch Phys Med Rehabil. 2012;93(2):221-28. [PMID:22289230] http://dx.doi.org/10.1016/j.apmr.2011.09.015

14. Canny ML, Thompson JM, Wheeler MJ. Reliability of the box and block test of manual dexterity for use with patients with fibromyalgia. Am J Occup Ther. 2009;63(4):506-10. [PMID:19708480] http://dx.doi.org/10.5014/ajot.63.4.506

15. Desrosiers JJ, Bravo GG, Hébert RR, Dutil EE, Mercier LL. Validation of the Box and Block Test as a measure of dexterity of elderly people: reliability, validity, and norms studies. Arch Phys Med Rehabil. 1994;75(7):751-55. [PMID:8024419] 
16. Hingtgen B, McGuire JR, Wang M, Harris GF. An upper extremity kinematic model for evaluation of hemiparetic stroke. J Biomech. 2006;39(4):681-88. [PMID:16439237] http://dx.doi.org/10.1016/j.jbiomech.2005.01.008

17. Dromerick AW, Schabowsky CN, Holley RJ, Monroe B, Markotic A, Lum PS. Effect of training on upper-extremity prosthetic performance and motor learning: A single-case study. Arch Phys Med Rehabil. 2008;89(6):1199-1204. [PMID:18503820] http://dx.doi.org/10.1016/j.apmr.2007.09.058

18. Miller LA, Stubblefield KA, Lipschutz RD, Lock BA, Kuiken TA. Improved myoelectric prosthesis control using targeted reinnervation surgery: A case series. IEEE Trans Neural Syst Rehabil Eng. 2008;16(1):46-50. [PMID:18303805] http://dx.doi.org/10.1109/TNSRE.2007.911817

19. Miller LA, Lipschutz RD, Stubblefield KA, Lock BA, Huang H, Williams TW 3rd, Weir RF, Kuiken TA. Control of a six degree of freedom prosthetic arm after targeted muscle reinnervation surgery. Arch Phys Med Rehabil. 2008;89(11):2057-65. [PMID:18996233] http://dx.doi.org/10.1016/j.apmr.2008.05.016

20. Jaspers E, Desloovere K, Bruyninckx H, Molenaers G, Klingels K, Feys H. Review of quantitative measurements of upper limb movements in hemiplegic cerebral palsy. Gait Posture. 2009;30(4):395-404. [PMID:19679479] http://dx.doi.org/10.1016/j.gaitpost.2009.07.110

21. Magermans DJ, Chadwick EK, Veeger HE, van der Helm FC. Requirements for upper extremity motions during activities of daily living. Clin Biomech (Bristol, Avon). 2005;20(6):591-99. [PMID:15890439] http://dx.doi.org/10.1016/j.clinbiomech.2005.02.006
22. Murgia A, Kyberd P, Barnhill T. The use of kinematic and parametric information to highlight lack of movement and compensation in the upper extremities during activities of daily living. Gait Posture. 2010;31(3):300-306.

[PMID:20034798] http://dx.doi.org/10.1016/j.gaitpost.2009.11.007

23. Fitoussi F, Diop A, Maurel N, Laassel M, Penneçot GF. Kinematic analysis of the upper limb: A useful tool in children with cerebral palsy. J Pediatr Orthop B. 2006;15(4): 247-56. [PMID:16751732] http://dx.doi.org/10.1097/01202412-200607000-00003

Submitted for publication October 24, 2013. Accepted in revised form March 4, 2014.

This article and any supplementary material should be cited as follows:

Hebert JS, Lewicke J, Williams TR, Vette AH. Normative data for modified Box and Blocks test measuring upper-limb function via motion capture. J Rehabil Res Dev. 2014;51(6):919-32.

http://dx.doi.org/10.1682/JRRD.2013.10.0228

ResearcherID/ORCID: Albert H. Vette, PhD: 0000-00022543-568X

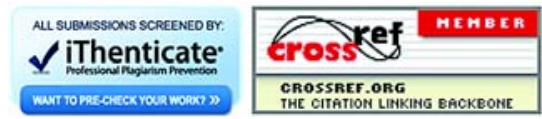


